\title{
THE NON-NILPOTENT GRAPH OF A SEMIGROUP
}

\author{
E. JESPERS AND M.H. SHAHZAMANIAN
}

\begin{abstract}
We associate a graph $\mathcal{N}_{S}$ with a semigroup $S$ (called the upper non-nilpotent graph of $S$ ). The vertices of this graph are the elements of $S$ and two vertices are adjacent if they generate a semigroup that is not nilpotent (in the sense of Malcev). In case $S$ is a group this graph has been introduced by A. Abdollahi and M. Zarrin and some remarkable properties have been proved. The aim of this paper is to study this graph (and some related graphs, such as the non-commuting graph) and to discover the algebraic structure of $S$ determined by the associated graph. It is shown that if a finite semigroup $S$ has empty upper non-nilpotent graph then $S$ is positively Engel. On the other hand, a semigroup has a complete upper non-nilpotent graph if and only if it is a completely simple semigroup that is a band. One of the main results states that if all connected $\mathcal{N}_{S}$-components of a semigroup $S$ are complete (with at least two elements) then $S$ is a band that is a semilattice of its connected components and, moreover, $S$ is an iterated total ideal extension of its connected components. We also show that some graphs, such as a cycle $C_{n}$ on $n$ vertices (with $n \geq 5$ ), are not the upper nonnilpotent graph of a semigroup. Also, there is precisely one graph on 4 vertices that is not the upper non-nilpotent graph of a semigroup with 4 elements. This work also is a continuation of earlier work by Okniński, Riley and the first named author on (Malcev) nilpotent semigroups.
\end{abstract}

\section{INTRODUCTION}

Malcev [17 and independently Neumann and Taylor [20] have shown that nilpotent groups can be defined by using semigroup identities (that is, without using inverses). This leads to the notion of a nilpotent semigroup (in the sense of Malcev). It was shown in [17, 20] (see also 21]) that a cancellative semigroup $S$ is nilpotent of class $n$ if and only if $S$ has a group of fractions which is nilpotent (in the classical sense) of class $n$. Properties of nilpotent semigroups have been studied by Lallement in [16], in particular he investigated the residual finiteness of finitely generated nilpotent regular semigroups (extending Hall's result on nilpotent groups). Meleshkin in [18] showed that free nilpotent semigroups are cancellative and Grigorchuk [12] showed that a finitely generated cancellative semigroup $S$ has finite GelfandKirillov dimension (or equivalently, its semigroup algebra $K[S]$ over a field

2010 Mathematics Subject Classification. Primary 20M07, 20M99, 05C25, Secondary: 20F18. Keywords and phrases: semigroup, nilpotent, graph.

Research partially supported by Onderzoeksraad of Vrije Universiteit Brussel, Fonds voor Wetenschappelijk Onderzoek (Belgium). 
$K$ has finite Gelfand-Kirillov dimension) if and only if $S$ is almost nilpotent (hence extending a celebrated result of Gromov). So in particular, finitely generated semigroup algebras of nilpotent semigroups have finite GelfandKirillov dimension.

In [13. Jespers and Okninski studied the prime images of semigroup algebras $K[S]$ of nilpotent semigroups $S$. It is shown that there is a close relationship with prime images of group algebras of nilpotent groups. The latter groups are closely related to the image of $S$ in the prime images of $K[S]$. Further the prime radical of $K[S]$ and the congruence it determines on $S$ are described. Also a full description of nilpotent semigroups of class 2 is given. It turns out that one obtains a complete analogue situation of the commutative case. In [14] it is described when the contracted semigroup algebra of a Malcev nilpotent semigroup is a prime Noetherian maximal order.

Jespers and Riley in [15] continued the investigations of nilpotent semigroups within the class of linear semigroups, i.e. subsemigroups of the multiplicative semigroup of all $n$-by- $n$ matrices over a field. For example, it is shown that the nilpotence of a linear semigroup can be characterized by a 4-generator semigroup condition, called the weakly Malcev nilpotent conditions (WMN). This can be considered as some kind of Engel's theorem for semigroups. Recall that Engel's famous theorem in Lie theory gives a certain 2-generator criterion for the global nilpotence of a finite-dimensional Lie algebra. It is also shown that a finitely generated residually finite group is nilpotent if and only if it is weakly Malcev nilpotent. Various other types of local and global nilpotence conditions are studied in [15], such as being positively Engel (PE) and Thue-Morse (TM). In each case necessary and sufficient conditions are proved for a linear semigroup to be of such a nilpotence type.

In the past twenty years fascinating questions and results have been raised and investigated by studying graphs associated to groups or rings (see for example [1, 2, 5, 6, 7, 9, 19, 23, 24]). In [1] the notion of a non-nilpotent graph $N_{G}$ of a group $G$ is introduced. The vertices of the graph are the elements of $G$ and there is an edge between vertices if they do not generate a nilpotent group. The authors studied the group $G$ by the information that is stored in this graph. Note that if the graph is empty (i.e. there are no edges) then every two-generated subgroup is nilpotent. In this case, if the group $G$ also is finite, then it is well known that $G$ is a nilpotent group. One of the results proved in [1] is that the number of connected components of $N_{G}$ for a finite group $G$ is either $\left|Z^{*}(G)\right|$ or $\left|Z^{*}(G)\right|+1$, where $Z^{*}(G)$ denotes the hypercenter of $G$. Note the elements of $Z^{*}(G)$ are isolated points in the graph $N_{G}$. Hence, in [1], one also studied the induced subgraph $\mathfrak{N}_{G}$ on $G \backslash \operatorname{nil}(G)$, where $\operatorname{nil}(G)$ is the subset of those elements $g \in G$ such that the group generated by $g$ and $h$ is nilpotent for any $h \in H$. In general it is unknown whether $\operatorname{nil}(G)$ is a subgroup of $\mathrm{G}$, but in many important cases it is. For example, $\operatorname{nil}(G)=Z^{\star}(G)$ if $G$ is a finite group (or more general, 
if $\mathrm{G}$ satisfies the maximal condition on its subgroups) or if $G$ is a finitely generated solvable group.

The aim of this paper is to study graphs associated to a semigroup and to discover the algebraic structure of the semigroup determined by its associated graph. The graphs of interest are those determined by the (non) nilpotence of two-generated subsemigroups. We mainly use notations as in [1]. Before stating our contributions, we first recall some definitions.

For a semigroup $S$ with elements $x, y, z_{1}, z_{2}, \ldots$ one recursively defines two sequences

$$
\lambda_{n}=\lambda_{n}\left(x, y, z_{1}, \cdots, z_{n}\right) \quad \text { and } \quad \rho_{n}=\rho_{n}\left(x, y, z_{1}, \cdots, z_{n}\right)
$$

by

$$
\lambda_{0}=x, \quad \rho_{0}=y
$$

and

$$
\lambda_{n+1}=\lambda_{n} z_{n+1} \rho_{n}, \quad \rho_{n+1}=\rho_{n} z_{n+1} \lambda_{n} .
$$

A semigroup is said to be nilpotent (in the sense of Malcev [17]) if there exists a positive integer $n$ such that

$$
\lambda_{n}\left(a, b, c_{1}, \cdots, c_{n}\right)=\rho_{n}\left(a, b, c_{1}, \cdots, c_{n}\right)
$$

for all $a, b$ in $S$ and $c_{1}, \cdots, c_{n}$ in $S^{1}$. The smallest such $n$ is called the nilpotency class of $S$. Clearly, null semigroups are nilpotent. As mentioned before (see for example [21]), a group $G$ is nilpotent of class $n$ if and only if it is nilpotent of class $n$ in the classical sense. In [13] it is proved that a completely (0-)simple semigroup $S$ is nilpotent if and only if $S$ is an inverse semigroup with nilpotent maximal subgroup $G$. Recall $([20])$ that a semigroup $S$ is said to be Neumann-Taylor (NT) if, for some $n \geq 2$,

$$
\lambda_{n}\left(a, b, 1, c_{2}, \cdots, c_{n}\right)=\rho_{n}\left(a, b, 1, c_{2}, \cdots, c_{n}\right)
$$

for all $a, b \in S$ and $c_{2}, \cdots, c_{n}$ in $S^{1}$. A semigroup $S$ is said to be positively Engel (PE) if, for some $n \geq 2$,

$$
\lambda_{n}\left(a, b, 1,1, c, c^{2}, \cdots, c^{n-2}\right)=\rho_{n}\left(a, b, 1,1, c, c^{2}, \cdots, c^{n-2}\right)
$$

for all $a, b$ in $S$ and $c \in S^{1}$.

Recall ([1]) that a semigroup $S$ is a completely 0-simple semigroup if and only if it is isomorphic with a regular Rees matrix semigroup over a group with zero, say $G^{0}$. The group $G$ is a maximal subgroup of $S$. The standard notation for such a semigroup $S$ is $\mathcal{M}^{0}(G, I, \Lambda ; P)$, where $I$ and $\Lambda$ are non-empty sets and $P$ is an $\Lambda \times I$ matrix with entries in $G^{0}=G \cup\{\theta\}$ (the latter is the group $G$ adjoined with a zero element $\theta$ ). The elements of $\mathcal{M}^{0}(G, I, \Lambda ; P)$ will be denoted as $g_{i j}$, where $g \in G^{0}, i \in I$ and $j \in \Lambda$. Note that all elements $\theta_{i j}$, with $i \in I, j \in \Lambda$, are identified with the zero element of $\mathcal{M}^{0}(G, I, \Lambda ; P)$, also denoted by $\theta$. If $P$ contains no zero entry then $\mathcal{M}^{0}(G, I, \Lambda ; P) \backslash\{\theta\}$ is a completely simple semigroup which is denoted as $\mathcal{M}(G, I, \Lambda ; P)$. 
As in [15], we denote by $F_{7}$ the semigroup which is the disjoint union of the completely 0 -simple semigroup $\mathcal{M}^{0}\left(\{e\}, 2,2 ; I_{2}\right)$ and the cyclic group $\{1, u\}$ of order 2 :

$$
F_{7}=\mathcal{M}^{0}\left(\{e\}, 2,2 ; I_{2}\right) \cup\{1, u\},
$$

where $I_{2}$ denotes the identity 2-by-2 matrix. The multiplication on $F_{7}$ is defined by extending that of the defining subsemigroups via $1 s=s 1=s$ for all $s \in \mathcal{M}^{0}\left(\{e\}, 2,2 ; I_{2}\right)$, and $e_{11} u=u e_{22}=e_{12}, e_{22} u=u e_{11}=e_{21}$. Note that

$$
F_{7}=\left\langle u, e_{11}\right\rangle \text {. }
$$

In [15] it is proved that a finite semigroup $S$ is positively Engel if and only if all non-null principal factors of $S$ are inverse semigroups whose maximal subgroups are nilpotent groups and $S$ does not have an epimorphic image that has $F_{7}$ as a subsemigroup.

Throughout the paper we will make frequently use of the above mentioned results, without specific reference.

In order to study (local) nilpotence or commutativity of semigroups, we define three types of graphs on a semigroup: the upper non-nilpotent graph $\mathcal{N}_{S}$, the lower non-nilpotent graph $\mathcal{L}_{S}$, and the non-commuting graph $\mathcal{M}_{S}$. In general, these graphs are different, however, we show that if any of these graphs is complete then so are the others. Moreover, in this case, it turns out that the semigroup $S$ is completely simple. We investigate which graphs can not show up as an upper non-nilpotent graph $\mathcal{N}_{S}$ for some semigroup $S$. In case $|S|<5$ then there is only one such graph. Our main results focus on the two extreme cases: (1) $\mathcal{N}_{S}$ is empty, that is every two-generated subsemigroup is nilpotent, and (2) the connected components are complete. The main results are the following:

(1) If $S$ is a semigroup, then $\mathcal{N}_{S}$ is complete if and only if $S$ is a completely simple semigroup that is a band. Moreover, $\mathcal{N}_{S}$ is complete if and only if $\mathcal{L}_{S}$ (or equivalently $\mathcal{M}_{S}$ ) is complete. Furthermore, for a finite semigroup $S$ of prime order, the graph $\mathcal{L}_{S}$ is connected if and only if it is complete.

(2) If $S$ is a semigroup such that all connected components of $\mathcal{N}_{S}$ are complete and have at least two elements then $S$ is a band and a semilattice of its connected components, in particular $S$ is semisimple. Moreover, if $S_{i}$ and $S_{j}$ are distinct connected components, then $S_{i} \cup S_{j}$ is a trivial total ideal extension of $S_{i}$ by $S_{j}$, or vice-versa, or $\left|S_{i} S_{j}\right|=1$.

(3) If $S$ is a finite semigroup such that $N_{S}$ is the empty graph, then $S$ is positively Engel.

(4) If $X$ is a graph with 4 vertices, then $X$ is the upper non-nilpotent graph of a semigroup if and only if $X \neq P_{4}$ (a path on 4 vertices).

(5) If $n \geq 5$ then $C_{n}$ (a graph which is a cycle on $n$ vertices) is not the upper non-nilpotent graph of any semigroup. 


\section{NON-NILPOTENT GRAPHS}

We begin by defining the graphs of interest on a semigroup $S$.

Definition 2.1. Let $S$ be a semigroup. The upper non-nilpotent graph $\mathcal{N}_{S}$ of a semigroup $S$ is the graph whose vertices are the elements of $S$ and in which there is an edge between two distinct vertices $x$ and $y$ if and only if the subsemigroup $\langle x, y\rangle$ generated by $x$ and $y$ is not a nilpotent semigroup.

The following lemma gives a criterion for a finite semigroup not to be nilpotent.

Lemma 2.2. A finite semigroup $S$ is not nilpotent if and only if there exists a positive integer $m$, elements $x, y \in S$ and $w_{1}, w_{2}, \cdots, w_{m} \in S^{1}$ such that $x=\lambda_{m}\left(x, y, w_{1}, w_{2}, \cdots, w_{m}\right), y=\rho_{m}\left(x, y, w_{1}, w_{2}, \cdots, w_{m}\right)$ and $x \neq y$ (note that for the converse one does not need that $S$ is finite).

Proof. Let $k=|S|$. If $S$ is not nilpotent then there exist elements $a, b \in S$ and some $w_{1}, \cdots, w_{k^{2}+1} \in S^{1}$ such that

$$
\lambda_{k^{2}+1}\left(a, b, w_{1}, \cdots, w_{k^{2}+1}\right) \neq \rho_{k^{2}+1}\left(a, b, w_{1}, \cdots, w_{k^{2}+1}\right) .
$$

Since $|S|^{2}=k^{2}$ there exist positive integers $t$ and $r \leq k^{2}+1, t<r$ with

$$
\begin{aligned}
& \left(\lambda_{t}\left(a, b, w_{1}, \cdots, w_{t}\right), \rho_{t}\left(a, b, w_{1}, \cdots, w_{t}\right)\right) \\
& \quad=\left(\lambda_{r}\left(a, b, w_{1}, \cdots, w_{r}\right), \rho_{r}\left(a, b, w_{1}, \cdots, w_{r}\right)\right) .
\end{aligned}
$$

Put $x=\lambda_{t}\left(a, b, w_{1}, \cdots, w_{t}\right), y=\rho_{t}\left(a, b, w_{1}, \cdots, w_{t}\right)$ and $m=r-t$. Then $x=\lambda_{m}\left(x, y, w_{t+1}, \cdots, w_{t+m}\right) \neq y=\rho_{m}\left(x, y, w_{t+1}, \cdots, w_{t+m}\right)$. This proves the necessity of the stated condition. That this condition is sufficient is obvious.

The lemma naturally leads us to the another graph on a semigroup.

Definition 2.3. The lower non-nilpotent graph $\mathcal{L}_{S}$ of a semigroup $S$ is the graph whose vertices are the elements of $S$ and there is an edge between two distinct vertices $x, y \in S$ if and only if there exist finitely many elements $w_{1}, w_{2}, \cdots, w_{n}$ in $\langle x, y\rangle^{1}$ such that $x=\lambda_{n}\left(x, y, w_{1}, w_{2}, \cdots, w_{n}\right)$ and $y=\rho_{n}\left(x, y, w_{1}, w_{2}, \cdots, w_{n}\right)$.

Clearly $\mathcal{L}_{S}$ is a subgraph of $\mathcal{N}_{S}$. In general these graphs are different. Indeed $F_{7}=\left\langle u, e_{11}\right\rangle$ (see (11)) is not positively Engel, and thus not nilpotent. So there is an edge between $e_{11}$ and $u$ and thus $\mathcal{N}_{F_{7}}$ is not empty. Since $\mathcal{M}^{0}\left(\{e\}, 2,2 ; I_{2}\right)$ and the cyclic group $\{1, u\}$ are nilpotent, there are no edges between elements of these semigroups in their respective lower non-nilpotent graphs. Further, because $\mathcal{M}^{0}\left(\{e\}, 2,2 ; I_{2}\right)$ is an ideal of $F_{7}$ it is impossible that for some positive integer $n$ one has that $u=\lambda_{n}\left(u, x, w_{1}, \cdots, w_{n}\right)$ for some $x \in \mathcal{M}^{0}\left(\{e\}, 2,2 ; I_{2}\right)$ and some $w_{1}, \cdots, w_{n} \in F_{7}$. Consequently, there is no edge in the graph $\mathcal{L}_{F_{7}}$ between $u$ and any element of $\mathcal{M}^{0}\left(\{e\}, 2,2 ; I_{2}\right)$. Similarly, there is no edge between 1 and any element of $\mathcal{M}^{0}\left(\{e\}, 2,2 ; I_{2}\right)$ in $\mathcal{L}_{F_{7}}$. Hence $\mathcal{L}_{F_{7}}$ is empty.

We now define a third graph; it contains $\mathcal{N}_{S}$ as a subgraph. 
Definition 2.4. The non-commuting graph $\mathcal{M}_{S}$ of a semigroup $S$ is the graph whose vertices are the elements of $S$ and in which there is an edge between two distinct vertices $x$ and $y$ if these elements do not commute.

Lemma 2.5. Let $S$ be a semigroup. Assume $\mathcal{N}_{S}$ is an empty graph. The following properties hold.

(1) Assume $m$ is a positive integer, $x, y \in S$ and $w \in S^{1}$. If $x=\lambda_{m}(x, y, w$, $w, \cdots, w)$ and $y=\rho_{m}(x, y, w, w, \cdots, w)$, then $x=y$.

(2) If an element of $S$ has an inverse, then this inverse is unique.

Proof. (1) Assume $x$ and $y$ are different elements of $S$ and $w \in S^{1}$ such that $x=\lambda_{m}(x, y, w, \cdots, w)$ and $y=\rho_{m}(x, y, w, \cdots, w)$ for some $m$. Then, we have $x w=\lambda_{m}(x w, y w, 1,1, \cdots, 1)$ and $y w=\rho_{m}(x w, y w, 1,1, \cdots, 1)$. As, by assumption $\mathcal{N}_{S}$ is empty, we get that $x w=y w$. Because of Lemma 2.2, we also get that $\langle x, y, w\rangle$ is not a nilpotent semigroup. Similarly, $w x=w y$. The equalities $w y=w x$, and $y w=x w$ and $y=\rho_{m}(x, y, w, w, \cdots, w)$ imply that $y \in\langle x, w\rangle$ and thus $\langle x, y, w\rangle=\langle x, w\rangle$. Hence $\langle x, w\rangle$ is not a nilpotent semigroup, a contradiction, because $\mathcal{N}_{S}$ is an empty graph.

(2) Let $a \in S$. Suppose $b$ and $c$ are inverses of $a$ in $S$. Then it is easily verified that $b=b a c a c a b$ and $c=c a b a b a c$. Hence $b=\lambda_{2}(b, c, a, a)$ and $c=$ $\rho_{2}(b, c, a, a)$. So, by part (1), $b=c$. Therefore $a$ has at most one inverse, as desired.

Clearly the lemma implies that every regular semigroup in which every two-generated semigroup is nilpotent is an inverse semigroup.

Theorem 2.6. Let $S$ be a finite semigroup. If every two-generated subsemigroup of $S$ is nilpotent (i.e. $\mathcal{N}_{S}$ is empty) then $S$ is positively Engel.

Proof. Assume $\mathcal{N}_{S}$ is an empty graph. Each principal factor of $S$ is either completely 0 -simple, completely simple or null. Every completely 0 -simple factor is isomorphic with a regular Rees matrix semigroup $S^{\prime}$ over a finite (maximal) subgroup $G$. Clearly, also $\mathcal{N}_{S^{\prime}}$ and $\mathcal{N}_{G}$ are empty graphs. Because of the former, Lemma 2.5 yields that $S^{\prime}$ is an inverse semigroup. The latter implies that every two-generated subgroup of $G$ is nilpotent and thus the finite group $G$ is nilpotent. Hence, $S^{\prime}$ is a nilpotent semigroup (see the introduction). Therefore, every non-null principal factor of $S$ is an inverse semigroup with maximal subgroup a nilpotent group.

Furthermore, we know that the two-generated semigroup $F_{7}$ is not nilpotent. As $\mathcal{N}_{S}$ is empty, it therefore follows that $F_{7}$ is not an epimorphic image of any subsemigroup of $S$. Therefore, $S$ is positively Engel.

It is well known that finite groups are positively Engel if and only if they are nilpotent [15]. However, in [15], it is shown that such a result is no longer true for finite semigroups. Indeed an example is given of a finite semigroup that is positively Engel but is not nilpotent. We now give another example: a finite semigroup with empty upper non-nilpotent graph (and thus it is positively Engel) but it is not nilpotent. 
Let $S$ be the semigroup that is the disjoint union $\mathcal{M}^{0}\left(\{e\}, 4,4 ; I_{4}\right) \cup\{w, v\}$, where $S^{\prime}=\mathcal{M}^{0}\left(\{e\}, 4,4 ; I_{4}\right)$ is a completely 0 -simple subsemigroup of $S$ that is an ideal of $S$ and such that the following relations are satisfied: $w^{2}=v^{2}=w v=v w=\theta, e_{11} w=e_{14}, e_{22} w=e_{23}, e_{33} w=\theta=e_{33} v, e_{44} w=\theta=$ $e_{44} v, e_{11} v=e_{13}, e_{22} v=e_{24}, w e_{11}=\theta=v e_{11}, w e_{22}=\theta=v e_{22}, w e_{33}=e_{23}$, $w e_{44}=e_{14}, v e_{33}=e_{13}$ and $v e_{44}=e_{24}$. We have $e_{31}=\lambda_{2}\left(e_{31}, e_{42}, w, v\right)$ and $e_{42}=\rho_{2}\left(e_{31}, e_{42}, w, v\right)$. Hence, by Lemma 2.2, the semigroup $S$ is not nilpotent. We now show that $\mathcal{N}_{S}$ is an empty graph. Since the semigroup $S^{\prime}$ is nilpotent, there are no edges between elements of $S^{\prime}$. Because the subsemigroup $\langle w, v\rangle$ is commutative, there is no edge between $w$ and $v$. Suppose now that there is an edge between $s \in S^{\prime}$ and $w$. Then, by Lemma 2.2, there exist elements $s_{1}, s_{2} \in\langle w, s\rangle$ and some elements $w_{1}, w_{2}, \cdots, w_{n} \in\langle w, s\rangle^{1}$ such that $s_{1}=\lambda_{n}\left(s_{1}, s_{2}, w_{1}, \cdots, w_{n}\right), s_{2}=\rho_{n}\left(s_{1}, s_{2}, w_{1}, \cdots, w_{n}\right)$ and $s_{1} \neq s_{2}$ (note that $s_{1} \neq \theta$ and $s_{2} \neq \theta$ ). Since $S^{\prime}$ is an ideal it follows that $s_{1}, s_{2} \in S^{\prime}$. As $S^{\prime}$ is a nilpotent semigroup, we furthermore obtain that at least one of the elements $w_{i}(1 \leq i \leq n)$ equals $w$. Now, if necessary, replacing $s_{1}$ by $\lambda_{i-1}\left(s_{1}, s_{2}, w_{1}, \cdots, w_{i-1}\right)$ and $s_{2}$ by $\rho_{i-1}\left(s_{1}, s_{2}, w_{1}, \cdots, w_{i-1}\right)$, we may assume that $w=w_{1}$. It then easily follows that $\theta \notin\left\{s_{1} w, w s_{1}, s_{2} w, w s_{2}\right\}$ and thus $\left\{s_{1}, s_{2}\right\} \subset\left\{e_{31}, e_{32}, e_{41}, e_{42}\right\}$. Also, $\theta \notin\left\{s_{1} w s_{2}, s_{2} w s_{1}\right\}$ and thus $\left\{s_{1}, s_{2}\right\}=\left\{e_{31}, e_{42}\right\}$. Suppose $s_{1}=e_{31}$ and $s_{2}=e_{42}$. Then $\lambda_{1}\left(s_{1}, s_{2}, w\right)=e_{32}$ and $\rho_{1}\left(s_{1}, s_{2}, w\right)=e_{41}$. Thus, $\theta \neq \lambda_{2}\left(s_{1}, s_{2}, w, w_{2}\right)=e_{32} w_{2} e_{41}$ and $\theta \neq$ $\rho_{2}\left(s_{1}, s_{2}, w, w_{2}\right)=e_{41} w e_{32}$. Hence, $w_{2} \notin S^{\prime}$ and thus $w_{2}=w$. But then $\theta \neq e_{32} w_{2} e_{41}=e_{32} w e_{41}=e_{33} e_{41}=\theta$, a contradiction. Similarly one shows that $s_{1}=e_{42}$ and $s_{2}=e_{31}$ leads to a contradiction. Hence we have shown that there are no edges between $w$ and any elements of $S^{\prime}$. Similarly, there are no edges between $v$ and elements of $S^{\prime}$. So, indeed, $\mathcal{N}_{S}$ is an empty graph.

One can improve the example a little in the sense that there exists a finite semigroup $T$ with empty upper non-nilpotent graph but $S$ is not NeumannTaylor. One can take for $T$ the previous example $S$ with an element $q$ added such that $S$ is a subsemigroup of $T$ and an ideal of $T$ and, moreover, it satisfies the relations $q^{2}=\theta, e_{11} q=e_{33} q=q e_{22}=q e_{44}=\theta, e_{22} q=e_{21}$, $e_{44} q=e_{43}, q e_{11}=e_{21}, q e_{33}=e_{43}, w q=e_{13}, v q=e_{23}, q w=e_{24}$ and $q v=e_{23}$. We leave the details to the reader.

\section{A Description of SEMigroups With COMPlete CONNECTED COMPONENTS FOR THE UPPER NON-NILPOTENT GRAPH}

In this section we give a description of semigroups for which all connected components of the upper non-nilpotent graphs are complete and contain at least two elements (so there are no isolated vertices). To do so, we first describe the semigroups that have complete upper non-nilpotent graphs. It turns out that this is equivalent with $\mathcal{L}_{S}\left(\right.$ or $\mathcal{M}_{S}$ ) being complete. We begin by showing that $\mathcal{L}_{S}$ is a complete graph provided that $\mathcal{L}_{S}$ is connected and $S$ is of prime order. 
We start with a technical lemma.

Lemma 3.1. Let $S$ be a semigroup. The following properties hold.

(1) If $I$ is an ideal of $S$ and $x \in S \backslash I$ then, in the graph $\mathcal{L}_{S}$, there is no edge between $x$ and any element of $I$.

(2) If an ideal I of $S$ intersects non-trivially a connected component of $\mathcal{L}_{S}$ then this connected component is contained in $I$.

In particular, all vertices of a connected component of $\mathcal{L}_{S}$ belong to the same J-class. Furthermore, if $\mathcal{L}_{S}$ is a connected graph, then $S$ is a simple semigroup.

Proof. (1) Suppose $I$ is an ideal of $S$ and $x \in S \backslash I$. Assume there is an edge in $\mathcal{L}_{S}$ between $y \in I$ and $x$. Then, $x=\lambda_{m}\left(x, y, z_{1}, \cdots, z_{m}\right)$ and $y=$ $\rho_{m}\left(x, y, z_{1}, \cdots, z_{m}\right)$ for some $z_{1}, \cdots, z_{m} \in S^{1}$ and some positive integer $m$. Since $I$ is an ideal of $S$, it is easily verified that then $x=\lambda_{m}\left(x, y, z_{1}, \cdots, z_{m}\right) \in I$, a contradiction.

(2) This follows easily from part (1).

Recall that a complete graph is a graph in which every pair of distinct vertices are connected with an edge.

Proposition 3.2. Let $S$ be a semigroup. The following properties hold.

(1) If $S$ is a completely simple semigroup that is a band, i.e. $S \cong$ $\mathcal{M}(\{e\}, I, \Lambda ; P)$, for some sets $I$ and $\Lambda$ and sandwich matrix $P$ all whose entries are $e$, then $\mathcal{L}_{S}$ is complete.

(2) If $S$ has prime order and $\mathcal{L}_{S}$ connected then $\mathcal{L}_{S}$ is complete.

Proof. (1) Let $e_{i j}$ and $e_{k l}$ be elements of $S=\mathcal{M}(\{e\}, I, \Lambda ; P)$. Then $e_{i j}=$ $\lambda_{2}\left(e_{i j}, e_{k l}, 1,1\right)$ and $e_{k l}=\rho_{2}\left(e_{i j}, e_{k l}, 1,1\right)$. Hence there is an edge between $e_{i j}$ and $e_{k l}$ in $\mathcal{L}_{S}$. Therefore, $\mathcal{L}_{S}$ is complete.

(2) Assume $S$ has prime order $p$ and $\mathcal{L}_{S}$ is connected. By Lemma 3.1, $S$ is a simple semigroup. Hence, $S$ is isomorphic with a regular Rees matrix semigroup over a (maximal) subgroup $G$, that is $S=\mathcal{M}(G, I, \Lambda ; P)$ for some sets $I$ and $\Lambda$. Since $|S|=p$, it follows that $S=G$ and $|S|=|G|=p$ or $G=\{e\}$. The former is excluded as a group of order $p$ is commutative and thus it has an empty lower non-nilpotent graph (and thus not a complete graph). Hence, $G=\{e\}$ and $\mathcal{L}_{S}$ is complete by part (1).

Note that if $\mathcal{L}_{S}$ is not connected then a $J$-class of $S$ may contain different connected components of $\mathcal{L}_{S}$. For example the simple semigroup $S=\mathcal{M}^{0}\left(\{1\}, 4,2 ;\left(\begin{array}{cccc}1 & 1 & \theta & \theta \\ \theta & \theta & 1 & 1\end{array}\right)\right)$ has only one $J$-class, but has edges only between $1_{11}, 1_{21}$ and $1_{32}, 1_{42}$ in $\mathcal{L}_{S}$.

We say that a vertex $v$ of a graph is totally connected if there are edges between $v$ and all other vertices of the graph.

Lemma 3.3. Let $S$ be a semigroup and $\mathcal{N}_{S}$ be its upper non-nilpotent graph. If a vertex a in $\mathcal{N}_{S}$ is totally connected, then a is idempotent. 
Proof. If $a^{2}=b$, then $\langle a, b\rangle=\left\langle a, a^{2}\right\rangle=\langle a\rangle$. Thus, $\langle a, b\rangle$ is nilpotent and there is no edge between $a$ and $b$. Which gives $a^{2}=a$.

Proposition 3.4. The following conditions are equivalent for a semigroup $S$.

(1) $\mathcal{L}_{S}$ is complete.

(2) $\mathcal{N}_{S}$ is complete.

(3) $\mathcal{M}_{S}$ is complete.

(4) $S$ is a completely simple semigroup that is a band, or equivalently, $S=\mathcal{M}(\{e\}, I, \Lambda ; P)$.

Proof. The implications (1) implies (2) and (2) implies (3) are obvious.

To prove (3) implies (4), assume $\mathcal{M}_{S}$ is complete. Then, since $x^{2} x=x x^{2}$ for $x \in S$, we get that $x$ is idempotent and thus $S$ is a band. Because $\mathcal{M}_{S}$ is complete, we have that each idempotent is primitive. As $S$ is band, we also obtain $a(a b a)=(a b a) a$ and $b(b a b)=(b a b) b$ for elements $a, b \in S$. The completeness of $\mathcal{M}_{S}$ implies that $a=a b a$ and $b=b a b$. Hence any two elements of $S$ are inverses of each other. Therefore, $S$ is completely simple that is a band (so its maximal subgroups are trivial). Equivalently, $S=\mathcal{M}(\{e\}, I, \Lambda ; P)$ with $P$ a sandwich matrix all whose components are equal to $e$. This proves (4).

That (4) implies (1) follows from Proposition 3.2.

Lemma 3.5. Let $S$ be a band. The following properties hold.

(1) $\mathcal{M}_{S}=\mathcal{N}_{S}$.

(2) Each complete connected component of $\mathcal{N}_{S}$ is a subsemigroup of $S$.

(3) If $\mathcal{N}_{S}$ has no isolated vertex, then each connected component of $\mathcal{N}_{S}$ is a subsemigroup of $S$.

Proof. (1) Let $x$ and $y$ be two arbitrary distinct elements of $S$. If $x y=y x$, then there is no edge between $x$ and $y$ in $\mathcal{N}_{S}$ nor in $\mathcal{M}_{S}$. If $x y \neq y x$, then $x y=\lambda_{2}(x y, y x, 1,1)$ and $y x=\rho_{2}(x y, y x, 1,1)$, because $x, y, x y, y x$ are idempotent elements. Then, by Lemma $2.2\langle x, y\rangle$ is not nilpotent. Hence there is an edge between $x$ and $y$ in both $\mathcal{N}_{S}$ and $\mathcal{M}_{S}$. Consequently, $\mathcal{M}_{S}=\mathcal{N}_{S}$.

(2) Suppose that $a, b \in S$ are in the same complete connected component of $\mathcal{N}_{S}$ but $c=a b$ is in a different connected component. As by the first part $\mathcal{M}_{S}=\mathcal{N}_{S}$, we have that $c$ commutes with $a$ and $b$. We then have $b a=(b a)(b a)=b(a b a)=b(c a)=b(a c)=b(a a b)=b(a b)=b c=c b=a b b=a b$. Therefore $a b=b a$, in contradiction with the fact there is an edge between $a$ and $b$ in $\mathcal{M}_{S}=\mathcal{N}_{S}$. Hence, indeed, each connected component of $\mathcal{N}_{S}$ is a subsemigroup.

(3) Suppose that $c, d \in S$ are in the same connected component of $\mathcal{N}_{S}$ but $e=c d$ is in a different connected component. As $\mathcal{N}_{S}$ has no isolated vertex, there exists an element $f \in S$ such that there is an edge between $e$ and $f$ in $\mathcal{N}_{S}$. By the first part, $f$ commutes with $c$ and $d$. Hence $f e=f c d=c f d=$ $c d f=e f$. This yields a contradiction with the fact that there is an edge between $e$ and $f$ in $\mathcal{N}_{S}=\mathcal{M}_{S}$. 
Note that if a band has an isolated vertex then in general its connected components are not subsemigroups. For example, let $B_{1}=\langle a, b\rangle$ and $B_{2}=$ $\langle a, c\rangle$ be two free bands and let $S$ be the semigroup that as a set is the union $B_{1} \cup B_{2} \cup\{\theta\}\left(\theta \notin B_{1} \cup B_{2}\right)$ and with multiplication such that $B_{1}$ and $B_{2}$ are subsemigroups, $\theta$ is a zero element and $x y=y x=\theta$ for $x \in B_{1} \backslash\langle a\rangle, y \in B_{2} \backslash\langle a\rangle$. Then the connected components of $S$ are $\{\theta\}$ and $S \backslash\{\theta\}$. Clearly the latter is not a subsemigroup.

It is easy to give an example of a finite semigroup $S$ such that $\mathcal{N}_{S} \neq \mathcal{M}_{S}$. Also one can easily construct a finite semigroup $T$ that is not a band and for which $\mathcal{N}_{T}=\mathcal{M}_{T}$. In general, even for a band $B$, one does not have that $\mathcal{L}_{B}=\mathcal{M}_{B}$.

In order to state the following result we recall (22]) that a total ideal extension of a semigroup $S$ by a semigroup $T$ is a semigroup $M$ that is the disjoint union $S \cup T$ and that contains $S$ and $T$ as subsemigroups such that $S$ is an ideal of $M$. If furthermore $s t=s=t s$ for all $s \in S$ and $t \in T$, then we call $M$ a trivial total ideal extension of $S$ by $T$ and we denote this by $T<S$. More generally, if $n>2$ then by $S_{1}<S_{2}<\cdots<S_{n}$ we denote the semigroup which is the disjoint union $\bigcup_{1 \leq i \leq n} S_{i}$ and for all $1 \leq i<j \leq n$, $S_{i} \cup S_{j}=S_{i}<S_{j}$. Or equivalently, $S_{1}<S_{2}<\cdots<S_{n}$ is defined recursively as $\left(S_{1}<S_{2}<\cdots<S_{n-1}\right)<S_{n}$.

Lemma 3.6. Let $S_{1}, S_{2}, \cdots, S_{n}$ be semigroups. There is no edge between any element of $S_{i}$ and any element of $S_{j}$ for $1 \leq i<j \leq n$ in $\mathcal{N}_{S_{1}}<S_{2}<\cdots<S_{n}$.

Proof. If $s \in S_{i}$ and $t \in S_{j}$ for $1 \leq i<j \leq n$, then $s t=t s$ because $S_{i} \cup S_{j}=$ $S_{i}<S_{j}$. Hence there is no edge between $s$ and $t$ in $\mathcal{N}_{S_{1}}<S_{2}<\cdots<S_{n}$.

We also recall another notion (see for example [11). Suppose $S$ is a semigroup such that $S=\bigcup\left\{S_{\alpha} \mid \alpha \in \Omega\right\}$, a disjoint union of subsemigroups $S_{\alpha}$, and such that for every pair of elements $\alpha, \beta \in \Omega$ we have $S_{\alpha} S_{\beta} \subseteq S_{\gamma}$ for some $\gamma \in \Omega$. One then has a product in $\Omega$ defined by $\alpha \beta=\gamma$ if $S_{\alpha} S_{\beta} \subseteq S_{\gamma}$ and one says that $S$ is the union of the band $\Omega$ of semigroups $S_{\alpha}$, with $\alpha \in \Omega$. If $\Omega$ is commutative, then one obtains a partial order relation $\leq$ on $\Omega$ with $\beta \leq \alpha$ if $\alpha \beta=\beta$. In this case $\Omega$ is a semilattice and one says that $S$ is the semilattice $\Omega$ of semigroups $S_{\alpha}$.

Theorem 3.7. Let $S$ be a semigroup and let $S_{\omega}$, with $\omega \in \Omega$, denote the connected $\mathcal{N}_{S}$-components. The following properties hold.

(1) If $\left|S_{\omega}\right|>1$, for each $\omega \in \Omega$, and if each connected $\mathcal{N}_{S}$-component is complete then $S$ is a band and each connected $\mathcal{N}_{S}$-component is a subsemigroup.

(2) If $S$ is a band and each connected $\mathcal{N}_{S}$-component is complete then $\mathcal{N}_{S}=\mathcal{M}_{S}$, the semigroup $S$ is a semilattice $\Omega$ of the semigroups $S_{\alpha}$, and either $S_{\alpha} \cup S_{\beta}$ is a trivial total ideal extension of $S_{\alpha}$ by $S_{\beta}$ or of $S_{\beta}$ by $S_{\alpha}$ or $S_{\alpha} S_{\beta}=S_{\alpha \beta}$ with $\left|S_{\alpha \beta}\right|=1$ for $\alpha, \beta \in \Omega$.

Proof. (1) Suppose $\left|S_{\omega}\right|>1$ for each $\omega \in \Omega$. Let $x_{1} \in S$ and put $x_{1}^{2}=x_{2}$. We need to prove that $x_{1}=x_{2}$. Assume the contrary. Since $\left\langle x_{1}, x_{2}\right\rangle$ is 
commutative, the elements $x_{1}$ and $x_{2}$ belong to different connected $\mathcal{N}_{S^{-}}$ components. Since the connected component containing $x_{2}$ has more than one element, there exists $x_{3} \in S$ such that there is an edge between $x_{2}$ and $x_{3}$ in $\mathcal{N}_{S}$. Note that $x_{1}$ and $x_{3}$ are then in different connected $\mathcal{N}_{S^{-}}$ components, and thus $\left\langle x_{1}, x_{3}\right\rangle$ is nilpotent. However, this yields a contradiction as $\left\langle x_{2}, x_{3}\right\rangle \subseteq\left\langle x_{1}, x_{3}\right\rangle$ and $\left\langle x_{2}, x_{3}\right\rangle$ is not nilpotent. Lemma 3.5 yields that each $S_{\alpha}$ is a semigroup.

(2) Suppose $S$ is a band and each connected $\mathcal{N}_{S}$-component is complete. By Lemma 3.5, $\mathcal{N}_{S}=\mathcal{M}_{S}$ and each $S_{\alpha}$ is a subsemigroup.

Fix $\alpha, \beta \in \omega$. Suppose $x_{1} \in S_{\alpha}$ and $x_{2} \in S_{\beta}$, with $\alpha \neq \beta$. So, $x_{1}$ and $x_{2}$ commute. Put $x_{3}=x_{1} x_{2}$. Then $x_{3}$ commutes with both $x_{1}$ and $x_{2}$. Let $\gamma \in \Omega$ be such that $x_{3} \in S_{\gamma}$. We claim that if $x_{3} \notin\left\{x_{1}, x_{2}\right\}$ then $\left|S_{\gamma}\right|=1$ and $S_{\alpha} S_{\beta}=S_{\gamma}$. Indeed, suppose that $x_{3} \notin\left\{x_{1}, x_{2}\right\}$. Because, by assumption, all connected $\mathcal{N}_{S}$-components are complete and $x_{1} x_{3}=x_{3} x_{1}, x_{2} x_{3}=x_{3} x_{2}$, we get that $x_{3} \notin S_{\alpha} \cup S_{\beta}$. So $x_{3} \in S_{\gamma}$ with $\gamma \notin\{\alpha, \beta\}$. By Proposition 3.4. $S_{\gamma}=\mathcal{M}(\{e\}, I, \Lambda ; P)$ with all entries of the sandwich matrix $P$ equal to $e$. Write $x_{3}=e_{i j}$ for some $i \in I$ and $j \in \Lambda$.

Assume $k \in I$ and let $x_{4}=e_{k j}$. Because $x_{1}$ and $x_{4}$ belong to different connected $\mathcal{N}_{S^{-}}$components, we have that $x_{1} x_{4}=x_{4} x_{1}$. Similarly, $x_{2} x_{4}=$ $x_{4} x_{2}$. Since also $x_{1}^{2}=x_{1}$ and $x_{4}^{2}=x_{4}$ we get that

$$
\begin{gathered}
x_{4} x_{3}=e_{k j} e_{i j}=x_{4}, \\
x_{1} x_{4}=x_{4} x_{1}=e_{k j} x_{1}=e_{k j} e_{i j} x_{1}=x_{4} x_{3} x_{1}=x_{4} x_{3}=e_{k j} e_{i j}=x_{4},
\end{gathered}
$$

and

$$
x_{2} x_{4}=x_{4} x_{2}=x_{4} x_{3} x_{2}=x_{4} x_{3}=x_{4} .
$$

Therefore,

$$
x_{3} x_{4}=x_{1} x_{2} x_{4}=x_{1} x_{4}=x_{4} .
$$

So $x_{4}$ and $x_{3}$ commute and they belong to the same complete connected $\mathcal{N}_{S}$-component. Therefore $x_{4}=x_{3}$ and thus $k=i$ and thus $|I|=1$. Similarly $|\Lambda|=1$ and thus $\left|S_{\gamma}\right|=1$.

Assume $a \in S_{\alpha}$. Because $S_{\alpha}$ is completely simple, we obtain from Proposition 3.4 that $S_{\alpha}=\mathcal{M}\left(\left\{e^{\prime}\right\}, I^{\prime}, \Lambda^{\prime} ; P^{\prime}\right)$ with all entries of the sandwich matrix $P^{\prime}$ equal to $e^{\prime}$. Write $x_{1}=e_{i^{\prime} j^{\prime}}^{\prime}$ and $a=e_{i^{\prime \prime} j^{\prime \prime}}^{\prime}$ for some $i^{\prime}, i^{\prime \prime} \in I$ and $j^{\prime}, j^{\prime \prime} \in \Lambda$. Let $a^{\prime}=e_{i^{\prime} j^{\prime \prime}}^{\prime} \in S_{\alpha}$. Then

$$
a a^{\prime}=a, a^{\prime} a=a^{\prime}, a^{\prime} x_{1}=x_{1}, x_{1} a^{\prime}=a^{\prime} .
$$

Note that we then also get that

$$
a^{\prime} a=x_{1} a .
$$

Since elements in different connected $\mathcal{N}_{S}$-components commute we get that

$$
x_{1} x_{2}=a^{\prime} x_{1} x_{2}=x_{1} x_{2} a^{\prime}=x_{2} x_{1} a^{\prime}=x_{2} a^{\prime}=a^{\prime} x_{2}=a^{\prime} a x_{2}=x_{1} a x_{2}=x_{1} x_{2} a .
$$

Thus we have proved that $a x_{1} x_{2}=x_{1} x_{2} a=x_{1} x_{2}$ for any $a \in S_{\alpha}$. Hence, for any $b \in S_{\alpha}$,

$$
a x_{1} b x_{2}=a x_{1} x_{2} b=x_{1} x_{2} b=b x_{1} x_{2}=x_{1} x_{2} .
$$


As $S_{\alpha}$ is a completely simple semigroup, we consequently obtain that $S_{\alpha} x_{2}=$ $\left\{x_{1} x_{2}\right\}$. Because $S_{\alpha}$ and $S_{\beta}$ commute, we get by symmetry that also $x_{1} S_{\beta}=$ $S_{\gamma}$ and thus $S_{\alpha} S_{\beta}=\left\{x_{1} x_{2}\right\}=S_{\gamma}$. This proves the claim.

So, for the remainder of the proof, we may assume that $x_{1} x_{2}=x_{2} x_{1} \epsilon$ $\left\{x_{1}, x_{2}\right\}$ for all $x_{1} \in S_{\alpha}$ and $x_{2} \in S_{\beta}$. Clearly we have that $\left\langle S_{\alpha} \cup S_{\beta}\right\rangle=S_{\alpha} \cup S_{\beta}$. We now show that either $S_{\alpha}$ or $S_{\beta}$ is an ideal in $\left\langle S_{\alpha} \cup S_{\beta}\right\rangle$. For if not, then, because of the symmetry in $S_{\alpha}$ and $S_{\beta}$, we may assume that there exist $x_{i} \in S_{\alpha}, x_{j}, x_{j^{\prime}} \in S_{\beta}$ with $x_{i} x_{j}=x_{i}, x_{i} x_{j^{\prime}}=x_{j^{\prime}}$. It follows that $x_{j} x_{j^{\prime}}=$ $x_{j} x_{i} x_{j^{\prime}}=x_{i} x_{j^{\prime}}=x_{j^{\prime}} x_{i}=x_{j^{\prime}} x_{i} x_{j}=x_{j^{\prime}} x_{j}$. So $x_{j}$ and $x_{j^{\prime}}$ distinct commuting elements in the connected connected $\mathcal{N}_{S}$-component $S_{\beta}$, in contradiction with the completeness of this component. Consequently, $S_{\alpha} \cup S_{\beta}$ is a trivial total ideal extension of $S_{\alpha}$ by $S_{\beta}$ or of $S_{\beta}$ by $S_{\alpha}$.

Corollary 3.8. Let $S$ be a finite semigroup and let $S_{i}$, for $1 \leq i \leq n$, denote the connected $\mathcal{N}_{S}$-components. If the connected component $S_{i}$ is complete and $\left|S_{i}\right|>1$ for all $1 \leq i \leq n$, then $S=S_{\sigma(1)}<S_{\sigma(2)}<\cdots<S_{\sigma(n)}$ for some $\sigma \in$ Sym $_{n}$.

Proof. Because of Theorem 3.7, we have $S_{i} \cup S_{j}=S_{i}<S_{j}$ or $S_{i} \cup S_{j}=S_{j}<S_{i}$ for $1 \leq i<j \leq n$. To prove the result it thus is sufficient to show that if $S_{i_{1}} \cup S_{i_{2}}=S_{i_{1}}<S_{i_{2}}$ and $S_{i_{2}} \cup S_{i_{3}}=S_{i_{2}}<S_{i_{3}}$ then $S_{i_{1}} \cup S_{i_{3}}=S_{i_{1}}<S_{i_{3}}$. Suppose this conclusion is false, i.e. assume that $S_{i_{1}} \cup S_{i_{3}}=S_{i_{3}}<S_{i_{1}}$. Then, for $s_{1} \in S_{i_{1}}, s_{2} \in S_{i_{2}}$ and $s_{3} \in S_{i_{3}}$ we get that $\left(s_{1} s_{2}\right) s_{3}=s_{2} s_{3}=s_{3}$ while $s_{1}\left(s_{2} s_{3}\right)=s_{1} s_{3}=s_{1}$, a contradiction.

If $S$ has an isolated vertex (i.e. $S$ has a connected $\mathcal{N}_{S}$-component with only one element) in $\mathcal{N}_{S}$, then the theorem does not hold in general. For example, all connected $\mathcal{N}_{S}$-components of the semigroup $S=\{a, b, c, d\}$ defined by the following Cayley table are complete but $S$ is not a band (the vertex $b$ is isolated and $\{a, c, d\}$ is a complete subgraph in $\mathcal{N}_{S}$.)

\begin{tabular}{c|cccc} 
& $a$ & $b$ & $c$ & $d$ \\
\hline$a$ & $b$ & $a$ & $c$ & $d$ \\
$b$ & $a$ & $b$ & $c$ & $d$ \\
$c$ & $d$ & $c$ & $c$ & $d$ \\
$d$ & $c$ & $d$ & $c$ & $d$
\end{tabular}

We have shown in Lemma 3.5 that in a band $S$ all complete connected $\mathcal{N}_{S^{-}}$ components are subsemigroups. In general this does not hold, for example if $S$ is an abelian semigroup then all connected $\mathcal{N}_{S}$-components are isolated and of course not necessarily subsemigroups. We do not know whether the connected components of the upper non-nilpotent graph of a semigroup are subsemigroups in case there are no isolated vertices. In this context we have the following remark.

Proposition 3.9. Let $S$ be a semigroup. If $\mathcal{N}_{S}$ does not have isolated vertices then, for every $x \in S$, the elements of the cyclic subsemigroup $\langle x\rangle$ are all in the same connected component. 
Proof. Suppose $x^{n} \in\langle x\rangle$ with $x^{n} \neq x$. Because $x$ and $x^{n}$ commute, there is no edge between them in $\mathcal{N}_{S}$. As, by assumption, $S$ does not have isolated vertices, there exists an element $y \in S$ such that there is an edge between $x^{n}$ and $y$ in $\mathcal{N}_{S}$. Clearly, $\left\langle x^{n}, y\right\rangle \subseteq\langle x, y\rangle$. Thus, $\langle x, y\rangle$ is not nilpotent and thus there is an edge between $x$ and $y$. Therefore $x$ and $x^{n}$ are in the same connected $\mathcal{N}_{S}$-component.

\section{Some Graphs are not UPPER NON-NilPotent GRAPhS}

For a positive integer $n$, we denote by $P_{n}$ a graph which is a path on $n$ vertices and by $C_{n}$ we denote a graph which is a unique cycle on $n$ vertices. A star graph $S_{n}$, is a tree with $n$ vertices such that one vertex (called the center) has degree $n-1$ and the other $n-1$ vertices (called the terminal vertices) have degree 1.

Let $S$ be a semigroup. In analogy with the group case [1, the set of vertices of the non-nilpotent graph of $S$ which are not adjacent to the vertex $x$, together with $x$, we call the nilpotentizer of $x$ in $S$. It will be denoted by $\operatorname{nil}_{S}(x)$. So

$$
\operatorname{nil}_{S}(x)=\{y \in S \mid\langle x, y\rangle \text { is nilpotent }\} .
$$

The nilpotentizer of $S$ is the set

$$
\operatorname{nil}(S)=\bigcap_{x \in S} \operatorname{nil}_{S}(x) .
$$

Thus $\operatorname{nil}(S)=\{x \in S \mid\langle x, y\rangle$ is nilpotent for all $y \in S\}$. Clearly, the center $Z(S)$ of $S$ is contained in nil $(S)$. The following lemma is easily verified.

Lemma 4.1. If $y \in n i l_{S}(x)$ and $z \in Z(S)$, then $y z \in n i l_{S}(x)$.

Again in analogy with the group case [1, we call a semigroup $S$ an $n$ semigroup if $\operatorname{nil}_{S}(x)$ is a subsemigroup of $S$ for every $x \in S$. In general, $\operatorname{nil}_{S}(x)$ is not a subsemigroup of $S$ for all $x \in S$. For example, nil ${ }_{S}\left(1_{11}\right)$ in the Rees matrix semigroup $\mathcal{M}^{0}\left(\{1\}, 2,3 ;\left(\begin{array}{cc}1 & \theta \\ \theta & 1 \\ 1 & \theta\end{array}\right)\right)$ is not a subsemigroup. Indeed the subsemigroups $\left\langle 1_{11}, 1_{12}\right\rangle$ and $\left\langle 1_{11}, 1_{23}\right\rangle$ are nilpotent, but $\left\langle 1_{11}, 1_{12} 1_{23}\right\rangle$ is not nilpotent.

If $X$ is a graph on at most three vertices then one can easily verify that $X$ is the upper non-nilpotent graph of a semigroup. If $|X|=4$ then this no longer holds, we show now that $P_{4}$ is the only exception. Recall that there are 126 semigroups with four elements.

Theorem 4.2. The graph $P_{4}$ is not the upper non-nilpotent graph of a semigroup with 4 elements.

Proof. We prove the result by contradiction. So, suppose $S=\{a, b, c, d\}$ is a semigroup such that its upper non-nilpotent graph is as depicted in Figure 1. The proof is given in six steps. In the first five steps we prove several 


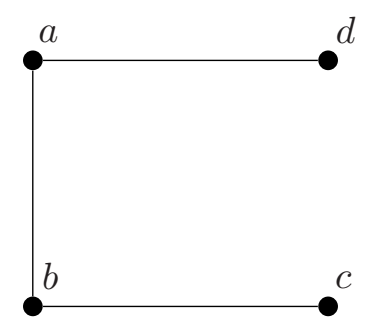

FiguRE 1. Graph $P_{4}$

restrictions that we may assume to hold in $S$. In step six we prove the final contradiction.

Step 1: $\langle c, d\rangle=\{c, d\},\langle a, c\rangle=\{a, c\},\langle b, d\rangle=\{b, d\}, c d=d c, a c=c a$, $b d=d b, c^{2}=c$ and $d^{2}=d$.

Because $\langle c, d\rangle$ is nilpotent and $\langle a, d\rangle$ is not nilpotent, it follows that $a \notin$ $\langle c, d\rangle$. Similarly $b \notin\langle c, d\rangle$. Hence $\langle c, d\rangle=\{c, d\}$. We now show that this semigroup is commutative. Indeed, for otherwise we may assume that $c d=c$ and $d c=d$. But $\lambda_{1}(c, d, 1)=c$ and $\rho_{1}(c, d, 1)=d$ and thus, by Lemma 2.2. $\langle c, d\rangle$ is not nilpotent, a contradiction. Similarly one obtains that $\langle a, c\rangle=$ $\{a, c\}, a c=c a$ and $\langle b, d\rangle=\{b, d\}, b d=d b$. Because $\langle a, d\rangle \nsubseteq\langle a, c\rangle$, we have that $c^{2} \neq d$ and thus $c^{2}=c$. Similarly $d^{2}=d$.

Step 2: $a c=c a=c, c d=d c=c, b d=d b=b, b \notin\{a d, d a\}$ and $a \notin\{b c, c b\}$.

We first note that $a c=c$ and $c d=d$ is not possible as it would imply $a d=a(c d)=(a c) d=c d=d c=d(c a)=(d c) a=d a$ and thus $\langle a, d\rangle$ is abelian, a contradiction. Similarly, it is not possible that $a c=a$ and $c d=c$. Thus we have that either $a c=c$ and $c d=c$, or $a c=a$ and $c d=d$. In the former case we obtain

$$
\begin{gathered}
c(a d)=(c a) d=(a c) d=c d=c=a c=a(c d)=a(d c)=(a d) c, \\
c(d a)=(c d) a=c a=c=d c=d(a c)=(d a) c
\end{gathered}
$$

and in the latter case one obtains $c(a d)=a d=(a d) c, c(d a)=d a=(d a) c$. So, in both cases, it follows that the elements $a d$ and $d a$ commute with $c$. As $\langle b, c\rangle$ is not nilpotent, it thus follows that $b \notin\{a d, d a\}$.

Similarly, we obtain that either $b d=b$ and $c d=c$, or $b d=d$ and $c d=d$. In both cases we also have $a \notin\{b c, c b\}$ (because there is an edge between $b$ and c).

As a result of the above, we are left with two remaining cases:

(1) $a c=c a=c, c d=d c=c, b d=d b=b, b \notin\{a d, d a\}$ and $a \notin\{b c, c b\}$,

(2) $a c=c a=a, c d=d c=d, b d=d b=d, b \notin\{a d, d a\}$ and $a \notin\{b c, c b\}$.

Because of symmetry reasons we only have to deal with the first case.

Step 3: $a \notin\langle b, c\rangle$ and $b \notin\langle a, d\rangle$.

We prove this by contradiction. So suppose $a \in\langle b, c\rangle$. Then there exists $\alpha \in S \backslash\{a\}$ such that $a=b \alpha$ or $a=c \alpha$. Both cases however are impossible as $\alpha \in\{b, c, d\}$ and $a \notin\{b c, c b\}, b d=b, b b \in\langle b, d\rangle, c d=c$ and $c c=c$. Hence the claim follows. Similarly, we also get that $b \notin\langle a, d\rangle$. 
Step 4: $\langle a, d\rangle=\{a, d\}, a d \neq d a$ and $a^{2}=a$.

Since $\langle a, d\rangle$ is not nilpotent, we obtain that there exist elements $w, v \in$ $\langle a, d\rangle^{1}$ such that $\lambda_{2}(a, d, w, v) \neq \rho_{2}(a, d, w, v)$. Because $b \notin\langle a, d\rangle$, we have that $\langle a, d\rangle \subseteq\{a, c, d\}$ and thus it follows that $\{a w d, d w a\}$ must be one of the following sets: $\{a, c\},\{d, c\}$ or $\{a, d\}$. In the first and second case, we get that $c \in\left\{\lambda_{1}(a, d, w, v), \rho_{1}(a, d, w, v)\right\}$. As $\langle a, d\rangle c=c\langle a, d\rangle=\{c\}$, it then follows that $\lambda_{2}(a, d, w, v)=\rho_{2}(a, d, w, v)=c$, a contradiction. Thus, we have $\{a w d, d w a\}=\{a, d\}$.

If $a w d=a$ (and thus $d w a=d$ ) then $a w=a$, because $c d=c$ and $d d=d$. Hence $a d=a$ and, clearly, $w \neq c$. If $w=1$ then also $d a=d$. If $w=a$, then also $a^{2}=a$ and $d a=d$. If $w=d$, then it is also clear that $d a=d$, because $d^{2}=d$. Hence, we have shown that $\{a d, d a\}=\{a, d\}$. Similarly, one can show that if $d w a=a$ then $\{a d, d a\}=\{a, d\}$.

Hence, we have proved that $\langle a, d\rangle=\{a, d\}$ and thus $\langle a, d\rangle=\{a d, d a\}=$ $\{a, d\}$ (as $\langle a, d\rangle$ is not nilpotent). It then follows that $a=a d=a(d a)=$ $(a d) a=a^{2}$ or $a=d a=(a d) a=a(d a)=a^{2}$. Thus, we have also $a^{2}=a$.

Step 5: $\langle b, c\rangle=\{b, c\}, b c \neq c b$ and $b^{2}=b$.

Because $\langle b, c\rangle$ is not nilpotent there exist elements $w, v \in\langle b, c\rangle^{1}$ such that $\lambda_{2}(b, c, w, v) \neq \rho_{2}(b, c, w, v)$. Because $a \notin\langle b, c\rangle$ we have that $\langle b, c\rangle \subseteq\{b, c, d\}$ and thus it follows that $\{b w c, c w b\}$ must be one of the sets: $\{b, d\},\{c, d\}$ or $\{b, c\}$.

If $\{b w c, c w b\}=\{b, d\}$ then $b w=b, w b=b$, because $\left\{d c, c d, c^{2}\right\}=\{c\}$. Hence $\{b c, c b\}=\{b w c, c w b\}=\{b, d\}$. Therefore we have two cases. If $b c=b$ and $c b=d$, then $(c b) c=d c=c$ and $c(b c)=c b=d$, a contradiction. If $b c=d$ and $c b=b$, then $(c b) c=b c=d$ and $c(b c)=c d=c$, a contradiction. So the case $\{b w c, c w b\}=\{b, d\}$ is excluded.

If $\{b w c, c w b\}=\{c, d\}$ then, because $\left\{d c, c d, c^{2}\right\}=\{c\}$, we get that $b w=b$ or $w b=b$. We claim that $d \in\{b c, c b\}$. Indeed, if $b w=w b$, then $\{b c, c b\}=$ $\{b w c, c w b\}=\{c, d\}$. On the other hand, if $b w \neq w b$, then, since $\langle b w, w b\rangle\langle b, c\rangle \subseteq$ $\{b, c, d\}$, we get that $b w=b$ and $w b \in\{c, d\}$ and thus $\{c, d\}=\{b w c, c w b\}=$ $\{b c, c\}$ and $d=b c$, or $w b=b$ and $b w \in\{c, d\}$ and we obtain similarly that $d=c b$. So this proves the claim. Now, since $\{b w c, c w b\}=\{c, d\}$ then $\{b w c v c w b, c w b v b w c\}=\{c v d, d v c\}$. If $v \in\{d, c, 1\}$ then

$$
\left\{\lambda_{2}(b, c, w, v), \rho_{2}(b, c, w, v)\right\}=\{b w c v c w b, c w b v b w c\}=\{c\},
$$

a contradiction. Thus $v=b$, and

$$
\left\{\lambda_{2}(b, c, w, v), \rho_{2}(b, c, w, v)\right\}=\{b w c v c w b, c w b v b w c\}=\{c b d, d b c\}=\{c b, b c\} .
$$

In particular $b c \neq c b$. Since $d \in\{b c, c b\} \subseteq\{b, c, d\}$, we also get that $b$ or $c$ belongs to $\{b c, c b\}$. If $b \in\{b c, c b\}$, then

$$
\left\{b w c b c w b, c w b^{3} w c\right\}=\{b w c v c w b, c w b v b w c\}=\{b c, c b\}=\{b, d\} .
$$

Thus $c w b^{3} w c \in\{b, d\}$ and therefore $c w b^{3} w c \neq c$. As $c^{2}=c$ and $c d=c$, we obtain that $w b^{3} w c=b$. Similarly because $d c=c$ and $c^{2}=c$, we get $c w b^{3} w=b$. Then $c w b^{3} w c=b c=c b$, a contradiction with $\langle b, c\rangle$ not being nilpotent. 
Otherwise if $c \in\{b c, c b\}$ then $\{b c, c b\}=\{c, d\}$ and is then readily verified that $(b c) b \neq b(c b)$, a contradiction. So also the case $\{b w c, c w b\}=\{c, d\}$ is excluded.

Finally, we deal with the remaining case $\{b w c, c w b\}=\{b, c\}$. We claim that $\{c b, b c\}=\{c, b\}$. Indeed recall that $w \in\langle b, c\rangle^{1}$ and $a \notin\langle b, c\rangle$. If $w \in$ $\{c, d, 1\}$, then we get $\{b, c\}=\{b w c, c w b\}=\{b c, c b\}$. If on the other hand $w=b$, then $b \in\left\{c b^{2}, b^{2} c\right\}$. Hence, because $c d=d c=c^{2}=c$, we get $b^{2}=b$. So again $\{b, c\}=\{b w c, c w b\}=\{c b, b c\}$. This proves the claim. Calculating $(b c) b=b(c b)$, it follows that $b^{2}=b$.

Step 6: The final contradiction.

Of course $a b \in\{a, b, c, d\}$. We show that each of the four possible cases leads to a contradiction.

First assume $a b=a$. Then $a d \neq d$, because otherwise $b=d b=a d b=a b=a$, a contradiction. As $\langle a, d\rangle=\{a, d\}$ and $a d \neq d a$, we then get that $a d=a, d a=$ $d$. But then, $d=d a=d a b=d b=b$, again a contradiction.

Second assume $a b=b$. Then $a d \neq a$, because otherwise $a b=b=b d=b d a=$ $b a$, in contradiction with $\langle a, b\rangle$ being non-commutative. Since $\langle a, d\rangle=\{a, d\}$ and since it is not nilpotent, we then get $a d=d, d a=a$. Hence $b=b d=$ $b(a d)=(b a) d$. Because we also know that $e d=d e=e$ for all $e \in S \backslash\{a\}$, it therefore follows that $b a \neq c, b a \neq d$. As $\langle b, a\rangle$ is non-commutative, we also get that $b a \neq b($ as $a b=b)$. Thus $b a=a$. But then $d=a d=b a d=b d=b$, a contradiction.

Third, assume $a b=c$. Recall that $\langle a, d\rangle=\{a d, d a\}=\{a, d\}$. If $a d=d$, then $c=a b=a d b=d b=b$, a contradiction. Hence $a d=a$ and $d a=d$. Consequently, $c=d c=d a b=d b=b$, again a contradiction.

Fourth, assume $a b=d$. Recall that $\langle b, c\rangle=\{b c, c b\}=\{b, c\}$. If $c b=b$, then $d=a b=a c b=c b=b$, a contradiction. Hence $c b=c$ and $b c=b$. Consequently, $c=d c=a b c=a b=d$, again a contradiction.

So we have reached in all possible cases a contradiction. Hence the result follows.

Note that the following Cayley table gives a semigroup $S$ with 5 elements such that its upper non-nilpotent graph has an induced subgraph on the set $\{b, c, d, e\}$ as in Figure 1:

\begin{tabular}{c|ccccc} 
& $a$ & $b$ & $c$ & $d$ & $e$ \\
\hline$a$ & $a$ & $a$ & $a$ & $a$ & $a$ \\
$b$ & $a$ & $b$ & $a$ & $a$ & $e$ \\
$c$ & $a$ & $a$ & $c$ & $d$ & $a$ \\
$d$ & $d$ & $d$ & $d$ & $d$ & $d$ \\
$e$ & $e$ & $e$ & $e$ & $e$ & $e$
\end{tabular}

Indeed $\langle b, d\rangle,\langle d, e\rangle$ and $\langle e, c\rangle$ are not nilpotent, but $\langle b, e\rangle,\langle b, c\rangle$ and $\langle d, c\rangle$ are nilpotent. Note however that $S \backslash\{a\}$ is not a subsemigroup of $S$.

In order to show that $P_{4}$ is the only graph that does not show as an upper non-nilpotent graph of a semigroup of order 4 , we now first give 
several classes of semigroups that are $n$-semigroups. In each case we include a specific example. It will follows that every graph on 4 vertices, except $P_{4}$, turns out to be the upper non-nilpotent graph of a semigroup on 4 elements that is of one of these types.

Example 4.3. The following classes of semigroups are all $n$-semigroups.

(1) Semigroups with empty upper non-nilpotent graph.

If $\mathcal{N}_{S}$ is an empty graph, then $\operatorname{nil}_{S}(x)=S$ for every $x \in S$. Of course examples of such semigroups are commutative semigroups.

(2) Semigroups with complete upper non-nilpotent graph.

If $\mathcal{N}_{S}$ is a complete graph then $\operatorname{nil}_{S}(x)=\{x\}$ for every $x \in S$. Because of Proposition 3.4, each element $x$ is idempotent and thus $S$ is an $n$-semigroup. Furthermore, an example of such a semigroup is a completely simple band $\mathcal{M}(\{e\}, n, 1 ; P)$.

(3) Semigroups whose upper non-nilpotent graph contains only one pair of non-adjacent vertices.

Suppose that $a$ and $b$ are the only elements of $S$ that are not connected by an edge. If $x \in S \backslash\{a, b\}$, then the vertex $x$ is totally connected and thus, by Lemma 3.3, $x$ is idempotent. Hence $\operatorname{nil}_{S}(x)=\{x\}$ is a subsemigroup. Because $\langle a, b\rangle$ is nilpotent and $\langle a, x\rangle$ is not nilpotent for $x \in S \backslash\{a, b\}$, we get that $x \notin\langle a, b\rangle$. Therefore $\langle a, b\rangle=\{a, b\}$ and also $\operatorname{nil}_{S}(a)=\operatorname{nil}_{S}(b)=\langle a, b\rangle$. Therefore $S$ is an $n$-semigroup. As an example one can take the semigroup $T$ that is the disjoint union of the semigroup $\mathcal{M}(\{e\}, n, 1 ; P)$ (with $n>1$ ) and the trivial group $\{1\}$ and such that the following relations are satisfied: $1 x=e_{11}$ and $x 1=x$ for every $x \in \mathcal{M}(\{e\}, n, 1 ; P)$. Only between 1 and $e_{11}$, there is no edge in $\mathcal{N}_{T}$. Indeed, by Proposition 3.4. $\mathcal{N}_{\mathcal{M}(\{e\}, n, 1 ; P)}$ is a complete subgraph. Since $1 e_{11}=e_{11} 1$, there is no edge between 1 and $e_{11}$. If $v \in \mathcal{M}(\{e\}, n, 1 ; P) \backslash\left\{e_{11}\right\}$, then $\left\langle e_{11}, v\right\rangle \subseteq\langle 1, v\rangle$. As $\left\langle e_{11}, v\right\rangle$ is not nilpotent, also $\langle 1, v\rangle$ is not nilpotent. Hence, there is an edge between $v$ and 1 in $\mathcal{N}_{\mathcal{M}(\{e\}, n, 1 ; P) \cup\{1\}}$.

(4) Semigroups such that their upper non-nilpotent graph is a disjoint union of a complete graph and one isolated vertex.

Indeed, suppose that $x$ is an isolated vertex. Of course the nilpotentizer of $x$ is $S$. If $y \in S \backslash\{x\}$ then $\operatorname{nil}_{S}(y)=\{x, y\}$. If $z \in S \backslash\{x, y\}$ then $\langle y, z\rangle$ is not nilpotent and $\langle x, y\rangle$ is nilpotent. Hence $z \notin\langle x, y\rangle$ and thus $\langle x, y\rangle \cap S \backslash\{x, y\}=\varnothing$. Therefore $\langle x, y\rangle=\{x, y\}$ and also nil ${ }_{S}(y)$ is a subsemigroup of $S$. Therefore, $S$ is an $n$-semigroup. An example of such a semigroup is $\mathcal{M}(\{e\}, n, 1 ; P)<S_{1}$ with $n>1$ and $\left|S_{1}\right|=1$. By Proposition 3.4. $\mathcal{N}_{\mathcal{M}(\{e\}, n, 1 ; P)}$ is a complete subgraph and by Lemma 3.6, there is

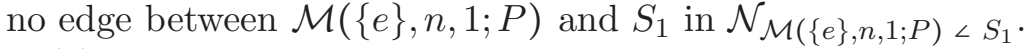

(5) Finite semigroups with complete connected upper non-nilpotent components such that each connected component has more than one element.

Because of Corollary 3.8 such a semigroup is of the form $S=S_{\sigma(1)}<\cdots<$ $S_{\sigma(n)}$, where $\sigma \in S y m_{n}$ and the connected components are $S_{\sigma(i)}, 1 \leq i \leq n$. Because of Theorem 3.7, $S$ is a band. If $x \in S_{\sigma(i)}$, then clearly nil ${ }_{S}(x)=$ 
$\{x\} \cup\left(S \backslash S_{\sigma(i)}\right)$. It can be easily verified that this is a subsemigroup of $S$. Therefore $S$ is an $n$-semigroup.

(6) Semigroups such that their upper non-nilpotent graph is a star graph.

If $a$ is the center of the graph $\mathcal{N}_{S}$ of such a semigroup then, by Lemma 3.3 . $a$ is idempotent. Hence, $\operatorname{nil}_{S}(a)=\{a\}$ is a subsemigroup. Between $a$ and any terminal element there is an edge. But between terminal elements of $S$, say $c$ and $d$, there is no edge. Hence $c d \neq a$, because otherwise $\langle a, c\rangle \subseteq\langle c, d\rangle$. This yields a contradiction as the former subsemigroup is not nilpotent while the latter is nilpotent. Therefore $S \backslash\{a\}$ is a subsemigroup and $S$ is an $n$ semigroup. An example of such a semigroup is $T_{n}=\left\{x_{0}, x_{1}, \cdots, x_{n}\right\}(n \geq 1)$ with multiplication defined by $x_{0} x_{i}=x_{0}$ and $x_{j} x_{i}=x_{1}$ for all $i$ and all $j \neq 0$. Since $x_{0} x_{1}=x_{0}, x_{1} x_{0}=x_{1}$, we have $x_{0}=\lambda_{1}\left(x_{0}, x_{1}, 1\right)$ and $x_{1}=\rho_{1}\left(x_{0}, x_{1}, 1\right)$ which implies the existence of an edge in $\mathcal{N}_{T_{n}}$ between $x_{0}$ and $x_{1}$. Moreover, since $\left\langle x_{1}, x_{0}\right\rangle \subseteq\left\langle x_{j}, x_{0}\right\rangle$ for $1 \leq j \leq n$ and $\left\langle x_{1}, x_{0}\right\rangle$ is not nilpotent, $\left\langle x_{j}, x_{0}\right\rangle$ is not nilpotent and there is an edge between $x_{0}$ and $x_{j}$ in $\mathcal{N}_{T_{n}}$. As $x_{j} x_{k}=$ $x_{k} x_{j}=x_{1}$ for $1 \leq j, k \leq n$ and $j \neq k$, there is no edge between $x_{k}$ and $x_{j}$ in $\mathcal{N}_{T_{n}}$. Therefore $\mathcal{N}_{T_{n}}$ is the star graph and its center is the element $x_{0}$.

(7) Semigroups such that their upper non-nilpotent graph is a disjoint union of a star graph and one isolated vertex.

Let $a$ be the center of the star subgraph of such a semigroup $S$ and let $c$ be an isolated vertex. If $b \notin\{a, c\}$ then $\langle a, b\rangle$ is not nilpotent while $\langle a, c\rangle$ is nilpotent. Hence $b \notin\langle a, c\rangle$. Therefore, $\operatorname{nil}_{S}(a)=\{a, c\}=\langle a, c\rangle$ is a subsemigroup. It can also be easily verified that the nilpotentizer of terminal elements of the star subgraph of $S$ are subsemigroups. Since also nil ${ }_{S}(c)=S$ is a semigroup we obtain that $S$ is an $n$-semigroup. An example of such a semigroup is $T_{n}<S_{1}$ with $\left|S_{1}\right|=1$.

(8) Semigroups such that their upper non-nilpotent graph is $C_{n}$ with $n \leq 4$.

In Theorem 4.6 it is shown that $n \geq 5$ can not occur. If $n \leq 3$, then $C_{n}$ is a complete graph and thus by (2) this semigroup is an $n$-semigroup. If $n=4$ then the statement is also easy to verify. The upper non-nilpotent graphs of the semigroups with the following Cayley tables

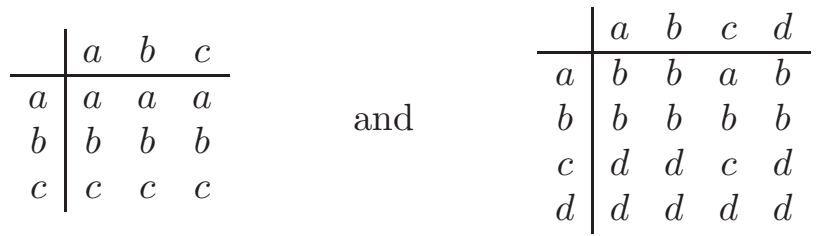

are $C_{3}$ and $C_{4}$ respectively.

Proposition 4.4. Let $X$ be a graph with at most 4 vertices. If $X \neq P_{4}$ then there exists a semigroup $S$ with $X$ as upper non-nilpotent graph. Moreover, all such semigroups are $n$-semigroups.

Proof. Let $X$ be a graph with at most 4 vertices. It can be easily verified that if $X$ is not as in one of the graphs given in Figure 2, then $X$ can be obtained as the non-nilpotent graph of one of the semigroup types given in 


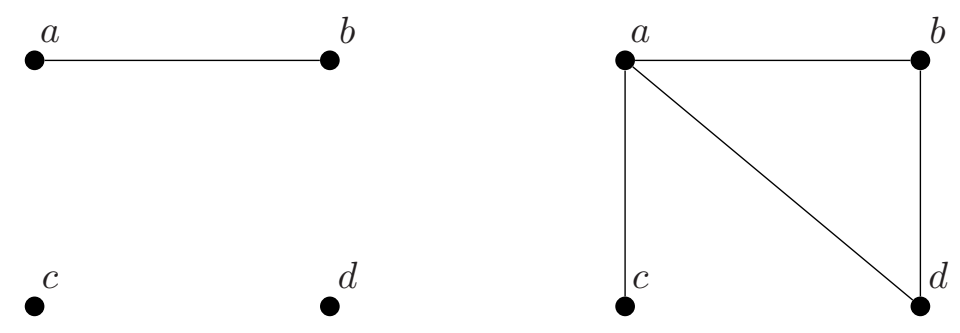

Figure 2.

Example 4.3. Furthermore, all semigroups with upper non-nilpotent graphs of one of these types are $n$-semigroups.

It can also be easily verified that the graphs in Figure 2 can be obtained as the upper non-nilpotent graphs of the following semigroups with respective Cayley tables

\begin{tabular}{c|cccc} 
& $a$ & $b$ & $c$ & $d$ \\
\hline$a$ & $a$ & $a$ & $a$ & $a$ \\
$b$ & $a$ & $b$ & $a$ & $a$ \\
$c$ & $a$ & $a$ & $c$ & $c$ \\
$d$ & $a$ & $a$ & $d$ & $d$
\end{tabular}

\begin{tabular}{l|llll} 
& $a$ & $b$ & $c$ & $d$ \\
\hline$a$ & $a$ & $a$ & $a$ & $a$ \\
$b$ & $b$ & $b$ & $b$ & $b$ \\
$c$ & $b$ & $b$ & $c$ & $d$ \\
$d$ & $d$ & $d$ & $d$ & $d$
\end{tabular}

These semigroups are $n$-semigroups. So it remains to show that all semigroups with upper non-nilpotent graphs as in Figure 2 are $n$-semigroups. Let $S=\{a, b, c, d\}$ be such a semigroup.

First we deal with the case when $\mathcal{N}_{S}$ is as the graph depicted on the right in Figure 2. Since $a$ is totally connected, $a$ is idempotent by Lemma 3.3 . Hence $\operatorname{nil}_{S}(a)=\{a\}$ is a subsemigroup. The subsemigroups $\langle a, b\rangle$ and $\langle b, d\rangle$ are not nilpotent, while $\langle b, c\rangle$ is nilpotent. Hence $a$ and $d$ are not in $\langle b, c\rangle$. Therefore, $\operatorname{nil}_{S}(b)=\{b, c\}=\langle b, c\rangle$ is a subsemigroup. Because $\langle a, d\rangle$ and $\langle b, d\rangle$ are not nilpotent, but $\langle c, d\rangle$ is nilpotent, we get that $a$ and $b$ are not in $\langle c, d\rangle$. Hence $\langle c, d\rangle=\{c, d\}$. Therefore $\operatorname{nil}_{S}(d)$ is a subsemigroup.

Since the order of both subsemigroups $\langle b, c\rangle$ and $\langle c, d\rangle$ is two and because these semigroups are nilpotent, one can easily verify that $c d=d c$ and $b c=c b$. We claim that $b d \neq a$. Indeed, because otherwise we have $a c=b d c=c b d=c a$, in contradiction with $\langle a, c\rangle$ not being nilpotent. Similarly $d b \neq a$. Hence $a \notin\{b d, d b\}$. As also $\langle b, c\rangle=\{b, c\}$ and $\langle c, d\rangle=\{c, d\}$, we obtain that nil ${ }_{S}(c)=$ $\{c, b, d\}$ is a subsemigroup. Therefore $S$ is an $n$-semigroup.

Next we deal with the case when $\mathcal{N}_{S}$ is the graph depicted on the left in Figure 2. We claim $c d \neq a$. We prove this by contraction. Assume $c d=a$. Because there is an edge between $a$ and $b$, but there is no edge between $b$ and $d$ and $c d=a, a$ and $c$ are not in $\langle b, d\rangle$. Hence $\langle b, d\rangle=\{b, d\}$ and since $\langle b, d\rangle$ is nilpotent, $b d=d b$. Also, as there is no edge between $a$ and $d$, we have that $b \notin\langle a, d\rangle$. Since $\langle b, d\rangle=\{b, d\}$, we have $d^{2}=d$. Similarly $\langle b, c\rangle=\{b, c\}$, $b c=c b, c^{2}=c$. Because $c$ and $d$ are idempotent and $c d=a$, we get $a d=a$ and $c a=a$. 
Because there is no edge between $c$ and $d$ and $c d=a$, one has $b \notin\langle d, c\rangle$. Hence $d c \neq b$. If $d c=d$, then $d c d=d$ and $d a=d$. Therefore $a=\lambda_{1}(a, d, 1)$ and $d=\rho_{1}(a, d, 1)$. Hence, by Lemma 2.2, $\langle a, d\rangle$ is not nilpotent, a contradiction. So $d c \neq d$ and, similarly, $d c \neq c$. Therefore $d c=a$. Because $c$ and $d$ are idempotent and $d c=a$, we have $d a=a$ and $a c=a$.

So we have $a c=c a=a d=d a=c d=d c=a, b d=d b$ and $b c=c b$. Consequently, $d b a=b d a=b a$ and $c b a=b c a=b a$. If $b a=c$ then $c=b a=d b a=d c=a$, a contradiction. If $b a=d$ then $d=b a=c b a=c d=a$, a contradiction too. Thus $b a \in\{a, b\}$. Similarly $a b \in\{a, b\}$. As there is an edge between $a$ and $b$, we have that $a b \neq b a$ and thus $\{a b, b a\}=\{a, b\}$.

Suppose $a b=a$ and $b a=b$. Then $d b=d b a=b d a=b a=b$ and $c b=c b a=$ $b c a=b a=b$ and thus $b=c b=c(d b)=(c d) b=a b=a$, a contradiction. Similarly, $a b=b$ and $b a=a$ lead to a contradiction.

This proves the claim that $c d \neq a$. Similarly $\{a, b\} \cap\langle c, d\rangle=\varnothing$. Consequently $\langle c, d\rangle=\{c, d\}$. It then can be easily verified from the graph of $S$ that $\operatorname{nil}_{S}(a)=\{a, c, d\}$ and $\operatorname{nil}_{S}(b)=\{b, c, d\}$ are subsemigroups. Therefore $S$ is an $n$-semigroup.

Corollary 4.5. If $X$ is a graph with 4 vertices then there exists a semigroup $S$ with 4 elements such that $X=\mathcal{N}_{S}$ if and only if $X \neq P_{4}$.

Proof. This follows immediately from Theorem 4.2. Example 4.3 and Proposition 4.4 .

Next we show that cycle graphs with at least 5 vertices can not be upper non-nilpotent graphs of semigroups.

Theorem 4.6. If $S$ is a finite semigroup of order $n \geq 5$ then $\mathcal{N}_{S} \neq C_{n}$.

Proof. Suppose that the semigroup $S=\left\{a_{1}, \cdots, a_{n}\right\}$ is such that $\mathcal{N}_{S}=C_{n}$, and there are edges between $a_{i}$ and $a_{i+1}$ for $1 \leq i \leq n$ (the addition used in the indices has to be interpreted modulo $n$ ).

First we show that $S$ is a band. Indeed, suppose $a_{i}^{2}=a_{j}$. Clearly $\left\langle a_{j+1}, a_{j}\right\rangle=\left\langle a_{j+1}, a_{i}^{2}\right\rangle \subseteq\left\langle a_{j+1}, a_{i}\right\rangle$ and $\left\langle a_{j-1}, a_{j}\right\rangle=\left\langle a_{j-1}, a_{i}^{2}\right\rangle \subseteq\left\langle a_{j-1}, a_{i}\right\rangle$. Because $\left\langle a_{j+1}, a_{j}\right\rangle$ and $\left\langle a_{j-1}, a_{j}\right\rangle$ are not nilpotent, we get that $\left\langle a_{j+1}, a_{i}\right\rangle$ and $\left\langle a_{j-1}, a_{i}\right\rangle$ are both not nilpotent. Hence $j=i$ and thus $S$ is band, because $n \geq 5$. Because of Lemma 3.5, we then have that $\mathcal{M}_{S}=\mathcal{N}_{S}$.

We claim that, $\left\langle a_{i}, a_{i+1}\right\rangle=\left\{a_{i}, a_{i+1}\right\}$. Since $n \geq 5$ there is no edge between $a_{i-2}$ and any of the elements $a_{i}$ and $a_{i+1}$. Hence, $a_{i-2}$ commutes with each of these elements and thus also with $a_{i} a_{i+1}$. It follows that $a_{i} a_{i+1} \notin\left\{a_{i-1}, a_{i-3}\right\}$.

If $n=5$ it then follows that $\left\{a_{i+1} a_{i}, a_{i} a_{i+1}\right\} \subseteq\left\{a_{i}, a_{i+1}, a_{i-2}\right\}$. If $a_{i} a_{i+1}=$ $a_{i-2}$, then $a_{i+1} a_{i} \in\left\{a_{i}, a_{i+1}\right\}$, because $a_{i+1} a_{i} \neq a_{i} a_{i+1}$. We suppose $a_{i+1} a_{i}=$ $a_{i+1}$. Then we have

$$
\begin{gathered}
a_{i-2} a_{i}=\left(a_{i} a_{i+1}\right) a_{i}=a_{i}\left(a_{i+1} a_{i}\right)=a_{i} a_{i+1}=a_{i-2}, \\
a_{i-2} a_{i+1}=a_{i+1} a_{i-2}=a_{i+1}\left(a_{i} a_{i+1}\right)=\left(a_{i+1} a_{i}\right) a_{i+1}=a_{i+1} a_{i+1}=a_{i+1} .
\end{gathered}
$$

Then

$$
a_{i+1}=a_{i-2} a_{i+1}=\left(a_{i-2} a_{i}\right) a_{i+1}=a_{i-2}\left(a_{i} a_{i+1}\right)=a_{i-2} a_{i-2}=a_{i-2},
$$


a contradiction. Similarly $a_{i+1} a_{i}=a_{i}$ yields a contradiction. Therefore $a_{i} a_{i+1} \in\left\langle a_{i}, a_{i+1}\right\rangle$. Similarly $a_{i+1} a_{i} \in\left\langle a_{i}, a_{i+1}\right\rangle$. Then $\left\langle a_{i}, a_{i+1}\right\rangle=\left\{a_{i}, a_{i+1}\right\}$.

Now we suppose that $n \geq 6$ and $a_{i} a_{i+1}=a_{j}$ such that $j \notin\{i, i+1\}$. Because $n \geq 6$, there exists element $a^{\star} \in\left\{a_{j+1}, a_{j-1}\right\}$ such that $a^{\star}$ is not adjacent to $a_{i}$ and to $a_{i+1}$. Which leads $a^{\star} a_{i} a_{i+1}=a_{i} a_{i+1} a^{\star}$. Therefore $a_{j} a^{\star}=a^{\star} a_{j}$, a contradiction. Then we have $\left\langle a_{i}, a_{i+1}\right\rangle=\left\{a_{i}, a_{i+1}\right\}$.

Since the subsemigroup $\left\langle a_{i}, a_{i+1}\right\rangle$ is not nilpotent, we thus obtain that $\left\{a_{i} a_{i+1}, a_{i+1} a_{i}\right\}=\left\{a_{i}, a_{i+1}\right\}$. We claim that if $a_{i} a_{i+1}=a_{i}$ then $a_{i+1} a_{i+2}=a_{i+1}$. Indeed, for otherwise we have

$$
a_{i} a_{i+1}=a_{i}, a_{i+1} a_{i}=a_{i+1}, a_{i+1} a_{i+2}=a_{i+2}, a_{i+2} a_{i+1}=a_{i+1} .
$$

Note that the elements $a_{i}$ and $a_{i+2}$ commute. Hence, we get

$$
\begin{gathered}
a_{i}=a_{i} a_{i+1}=a_{i} a_{i+2} a_{i+1}=a_{i+2} a_{i} a_{i+1}=a_{i+2} a_{i}= \\
a_{i+1} a_{i+2} a_{i}=a_{i+1} a_{i} a_{i+2}=a_{i+1} a_{i+2}=a_{i+2},
\end{gathered}
$$

a contradiction. This proves the claim.

Suppose now that $a_{i} a_{i+1}=a_{i}$. Since $a_{i} a_{i+1}=a_{i}$, we have from the above that $a_{i+1} a_{i+2}=a_{i+1}$ and so $a_{i} a_{i+2}=a_{i} a_{i+1} a_{i+2}=a_{i} a_{i+1}=a_{i}$. Because $a_{i} a_{i+1}=$ $a_{i}, a_{i+1} a_{i+2}=a_{i+1}, a_{i} a_{i+1} \neq a_{i+1} a_{i}, a_{i+1} a_{i+2} \neq a_{i+2} a_{i+1},\left\langle a_{i}, a_{i+1}\right\rangle=\left\{a_{i}, a_{i+1}\right\}$ and $\left\langle a_{i+1}, a_{i+2}\right\rangle=\left\{a_{i+1}, a_{i+2}\right\}$, we get that $a_{i+1} a_{i}=a_{i+1}$ and $a_{i+2} a_{i+1}=a_{i+2}$. Hence, $a_{i+2} a_{i}=a_{i+2} a_{i+1} a_{i}=a_{i+2} a_{i+1}=a_{i+2}$ and thus $\left\langle a_{i}, a_{i+2}\right\rangle=\left\{a_{i}, a_{i+2}\right\}$.

Similarly if $a_{i} a_{i+1}=a_{i+1}$, we have $\left\langle a_{i}, a_{i+2}\right\rangle=\left\{a_{i}, a_{i+2}\right\}$.

The above information shows that $\left\{a_{i}, a_{i+1}, a_{i+2}\right\}$ is a subsemigroup. Since $a_{i}=\lambda_{1}\left(a_{i}, a_{i+1}, 1\right), a_{i+1}=\rho_{1}\left(a_{i}, a_{i+1}, 1\right)$ or $a_{i}=\lambda_{2}\left(a_{i}, a_{i+1}, 1,1\right), a_{i+1}=\rho_{2}\left(a_{i}\right.$, $\left.a_{i+1}, 1,1\right)$, there is an edge between $a_{i}$ and $a_{i+1}$ in $\mathcal{L}_{\left\{a_{i}, a_{i+1}, a_{i+2}\right\}}$. Similarly there is an edge between $a_{i+1}$ and $a_{i+2}$ in $\mathcal{L}_{\left\{a_{i}, a_{i+1}, a_{i+2}\right\}}$. But there is no edge between $a_{i}$ and $a_{i+2}$ in this graph, because they commute. As the the order of the semigroup $\left\{a_{i}, a_{i+1}, a_{i+2}\right\}$ is of prime order and the lower nonnilpotent graph of this semigroup is connected but not complete this yields a contradiction with Proposition 3.2 .

Note that $P_{4}$ and $C_{5}$ are isomorphic to their respective complements. So a question of interest is whether a graph that is isomorphic to its complement graph can occur as an upper non-nilpotent graph of a semigroup. Note that $C_{n}$ (expect $n=5$ ) is not isomorphic to its complement graph.

Acknowledgment The authors would like to thank the referee for a thorough report that resulted in a much improved version of the paper.

\section{REFERENCES}

[1] A. Abdollahi and M. Zarrin, Non-nilpotent graph of a group, Comm. Algebra 38 (12) (2010) 4390-4403.

[2] A. Abdollahi, Engle graph associated to a group, J. Algebra 318 (2) (2007) 680-691.

[3] A. Abdollahi, S. Akbari and H.R. Maimani, Non-commuting graph of a group, J. Algebra 298 (2006) 468-492.

[4] S. Akbari, H.R. Maimani, and S. Yassemi, When a zero-divisor graph is planar or complete r-partite graph, J. Algebra 270 (2003) 169-180. 
[5] S. Akbari and A. Mohammadian, On the zero-divisor graph of a commutative ring, J. Algebra 274 (2) (2004) 847-855.

[6] D.F. Anderson and P.S. Livingston, The zero-divisor graph of a commutative ring, J. Algebra 217 (1999) 434-447.

[7] I. Beck, Coloring of commutative rings, J. Algebra 116 (1988) 208-226.

[8] E.A. Bertram, Some applications of graph theory to finite groups, Discrete Math. 44 (1983) 31-43.

[9] E.A. Bertram, M. Herzog, A. Mann, On a graph related to conjugacy classes of groups, Bull. London Math. Soc. 22 (6) (1990) 569-575.

[10] J.A. Bondy and U.S.R. Murty, Graph theory with applications, Elsevier Science Publishing Co. Inc. (1976).

[11] A.H. Clifford and G.B. Preston, The Algebraic Theory of Semigroups, American 35 Mathematical Society Providence (1961).

[12] R.I. Grigorchuk, Cancellative semigroups of power growth, Mat. Zametki 43 (1988) 305-319 (In Russian).

[13] E. Jespers and J. Okniński, Nilpotent semigroups and semigroup algebras, J. Algebra 169 (3) (1994) 984-1011.

[14] E. Jespers and J. Okniński, Semigroup algebras and maximal orders, Canadian Math. Bulletin 42 (3) (1999) 298-306.

[15] E. Jespers and D. Riley, Nilpotent Linear Semigroups, International Journal of Algebra and Computation 16 (1) (2006) 1-20.

[16] G. Lallement, On nilpotency in semigroups, Pacific J. Math. 42 (1972) 693-700.

[17] A. I. Malcev, Nilpotent semigroups, Uc. Zap. Ivanovsk. Ped. Inst. 4 (1953) 107-111 (in Russian).

[18] A.V. Meleshkin, Regular semigroups of polynomial growth, Mat. Zametki 47 (2) (1990) 58-64.

[19] B.H. Neumann, A problem of Paul Erdös on groups, J. Aust. Math. Soc. Ser. A 21 (1976) 467-472.

[20] B.H. Neumann, Tekla Taylor, Subsemigroups of nilpotent groups, Proc. Roy. Soc, Ser. A 274 (1963) 1-4.

[21] J. Okniński, Semigroup Algebras, Marcel Dekker, New York, (1981).

[22] B. Steinberg, The q-theory of finite semigroups, Ann. of Math, 1 (1968).

[23] Y. Segev, The commuting graph of minimal nonsolvable groups, Geom. Dedicata 88 (2001) 55-66.

[24] J.S. Williams, Prime graph components of finite groups, J. Algebra 69 (2) (1981) $487-513$.

Department of Mathematics, Vrije Universiteit Brussel, Pleinlaan 2, 1050 Brussel, Belgium

E-mail address: ef jesper@vub.ac.be, m.h.shahzamanian@vub.ac.be 